\title{
Abnormal intrafusal muscle fibres in myotonic dystrophy: a study using serial sections
}

\author{
M. SWASH AND K. P. FOX \\ From the Section of Neurological Sciences, The London Hospital, London
}

SYNOPSIS Studies of the extent and complexity of intrafusal muscle fibre splitting in myotonic dystrophy have been carried out in three spindles obtained at muscle biopsy, and in nine spindles found in the lumbrical muscle of a case that came to necropsy. It is suggested that splitting, due to mechanical stresses, leads to a regenerative response in the separated fragments.

In myotonic dystrophy a characteristic abnormality occurs in the muscle spindles. Affected spindles contain large numbers of abnormally thin intrafusal muscle fibres, usually arranged in several groups within the spindle lumen. In addition, changes occur in both the motor and the sensory innervation, and the spindle capsule is usually thickened.

It has been suggested that the abnormal intrafusal muscle fibres are formed by longitudinal splitting of parent fibres (Daniel and Strich, 1964; Swash, 1972), but the pathogenesis of this splitting, which may occur in muscles in which the extrafusal fibres are almost normal, is not understood. The abnormal intrafusal muscle fibres are often so thin that, in serial sections of paraffin-embedded muscles, it is not usually possible to identify individual fibres. Consequently, the longitudinal extent of the abnormality has not been studied.

We have examined this problem by the method of serial transverse section. Twelve spindles have been studied; three were obtained by muscle biopsy and nine were found in the paraffin-embedded lumbrical muscle of a patient reported previously (Swash, 1972). Our findings will be discussed in relation to the pathogenesis of the spindle abnormality in this disease.

\section{METHODS}

A woman, aged 52 years, presented with typical, moderately advanced myotonic dystrophy. Distal, cervical, and sternomastoid muscles were pre(Accepted 8 August 1974). dominantly affected and myotonia was present, both clinically and electromyographically, in the whole skeletal musculature. Bilateral cataracts were present, and there was frontal baldness and a mild degree of mental retardation. There were several affected relatives. Consent was given for muscle biopsy.

The pronator teres muscle, which was clinically affected, was chosen for biopsy, since Cooper (1966) has shown that this muscle contains a large number of muscle spindles. The biopsy specimen was divided into three pieces. One portion was pinned onto stiff card and fixed in $10 \%$ formol-saline for light microscopy, and another was quenched in hexane, which had been cooled with a freezing mixture, and was prepared for study using routine enzyme histochemical methods (Dubowitz and Brooke, 1973). A third portion was cut into small pieces and fixed for four hours in $2.5 \%$ barbiturate-buffered glutaraldehyde at $4{ }^{\circ} \mathrm{C}$, post-fixed in $2 \%$ phosphate-buffered osmium tetroxide, dehydrated in a series of graded alcohols, and embedded in Araldite.

One spindle was found in the frozen blocks examined by enzyme histochemical methods and two were found in the Araldite-embedded material. The latter were examined in 1 or $2 \mu \mathrm{m}$ thick, serial sections stained with toluidine blue (more than 600 sections). Photomicrographs of one spindle were made at a magnification of 350 diameters, at 5 or $10 \mu \mathrm{m}$ intervals. These were mounted in series on large cards and examined with a hand lens, in order to study the longitudinal extent of any 'splitting' of intrafusal muscle fibres.

At selected levels thin transverse sections were mounted on Formvar-coated, $1.0 \mathrm{~mm}$ diameter, single aperture grids, stained with uranyl acetate and lead citrate by standard methods, and examined with 
an AE1 6B electron microscope at $60 \mathrm{kV}$. The fine structure of these spindles was compared with that of normal human and baboon muscle spindles (unpublished observations) and with that of spindles of other mammals (Landon, 1966; Banker and Girvin, 1971; Barker et al., 1972; Smith and Ovalle, 1972).

In addition, serial $6 \mu \mathrm{m}$ transverse sections were cut through the paraffin-embedded first lumbrical muscle of one, previously studied case, that came to necropsy, aged 18 years (see Swash, 1972). In these sections, stained with haematoxylin and eosin, nine spindles were examined through most of their length.

\section{RESULTS}

SERIAL SEMI-THIN SECTIONS Two muscle spindles were studied in serial $2 \mu \mathrm{m}$ thick sections stained with toluidine blue. Serial sections of one spindle extended from the mid-polar region of one pole, through the equatorial region, to the juxtaequatorial region of the opposite pole. In the mid-polar sections of this spindle there were more than 100 abnormally thin intrafusal muscle fibres: the number found varied greatly even in sections $10 \mu \mathrm{m}$ apart. The diameter of these abnormal fibres ranged from less than $1 \mu \mathrm{m}$ to $7 \mu \mathrm{m}$, but most were narrower than $2 \mu \mathrm{m}$. They were usually arranged in clusters of three to 30 fibres.

Study of the serial sections showed that this abnormality was the result both of longitudinal splitting and of fusion of individual fibres within a given cluster. This process occurred along the length of the pole of the spindle (Fig. 1a-d). It was so complex that it was often difficult to identify a single abnormal fibre in sections separated by only a few micra. Each abnormal fibre therefore represented a fragment separated from its adjacent fibres for only a short part of its length. Nuclei were found in some, but not in all, of these fragments. There was great variation in the staining intensity of individual fragments of intrafusal fibres even within a single cluster (Fig. 1). Several ring fibres were observed. Each surrounded a cluster of abnormal fibres, or passed diagonally between the fibres of a single cluster toward its circumference.

The sections of the second spindle included the tip of its pole. In this region there were four normal intrafusal muscle fibres. In sections cut $50 \mu \mathrm{m}$ deeper into the block three of these fibres had become split into three or more abnormal fibres. In the subsequent serial sections this process became more complex until the abnormality was similar to that found in the other spindle.

In both spindles the abnormality became less complex as the equator was approached and, in the equatorial sensory region itself, longitudinal splitting of the intrafusal fibres was far less prominent (Fig. 1d). Many of the fibres remained smaller than normal but several clusters of fibres formed bag or chain aggregations of nuclei in single, undivided, fibres of normal diameter (Fig. 2). The nuclear aggregations were not as prominent and the nuclei were often not as tightly packed, however, as in normal spindles sectioned in this region. In these abnormal spindles the primary sensory region was unusually short $(20-50 \mu \mathrm{m})$, but the sensory endings themselves were normal (Fig. 2). The region between the pole and the equatorial sensory region, in which fusion of abnormal fibres occurred, was approximately $50 \mu \mathrm{m}$ in length.

There were only slight changes in the extra- $\vec{D} \overrightarrow{0}$ fusal muscle fibres. Some contained centralo nuclei. There were several hypertrophied fibres $\frac{\rho}{6}$ 을 and, rarely, part of the edge of a fibre was split $\stackrel{-}{-}$ into several smaller fragments (see Schröder g and Adams, 1968). A few small, degenerateo 일 fibres were present.

ENZYME HISTOCHEMISTRY In polar sections of the single spindle found in the blocks of frozen tissue there were five normal intrafusal muscle fibres. One abnormal fibre appeared split into 11 tiny fragments which were all of the same histochemical type (Fig. 3). All the intrafusal fibres showed prominent NADH tetrazolium reductase activity and relative unreactivity for phosphorylase. The reaction for myofibrillar ATPase after preincubation at $\mathrm{pH} 9.5$ demonstrated strongly reactive, intermediate, and weakly reactive fibres. This variation in the ATPase reaction occurs in normal human intrafusal muscle fibres (Spiro and Beilin, 1969).

ELECTRON MICROSCOPY Each cluster of intrafusal muscle fibres was surrounded by a layer of basement membrane and, usually, by a thin cytoplasmic layer derived from intraluminal invaginations of capsular cells or fibrocytes. Mid polar sections contained the largest number of clusters. At all sectional levels there were 


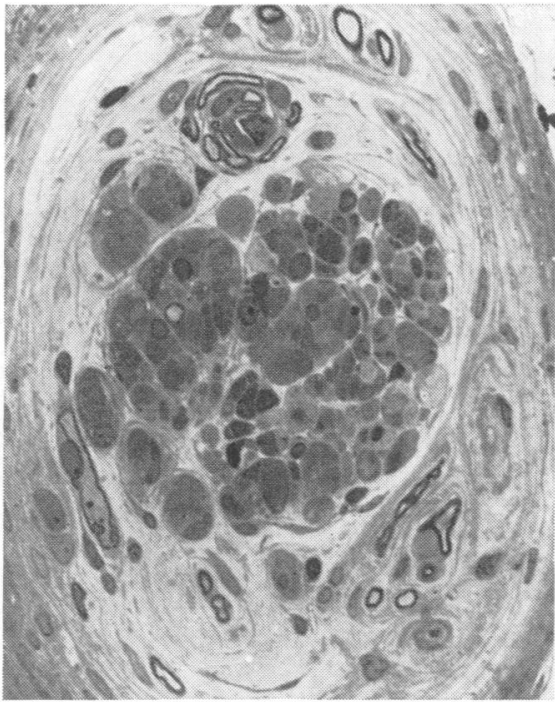

(a)

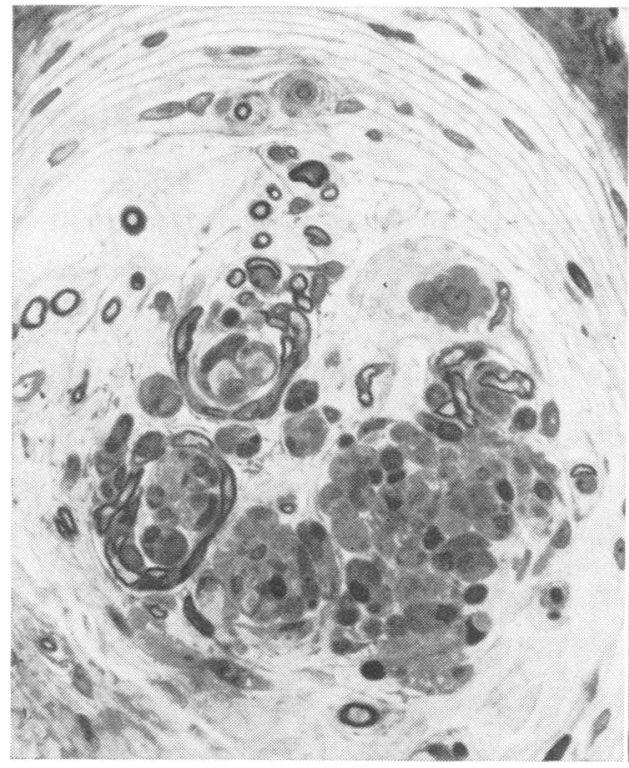

(c)

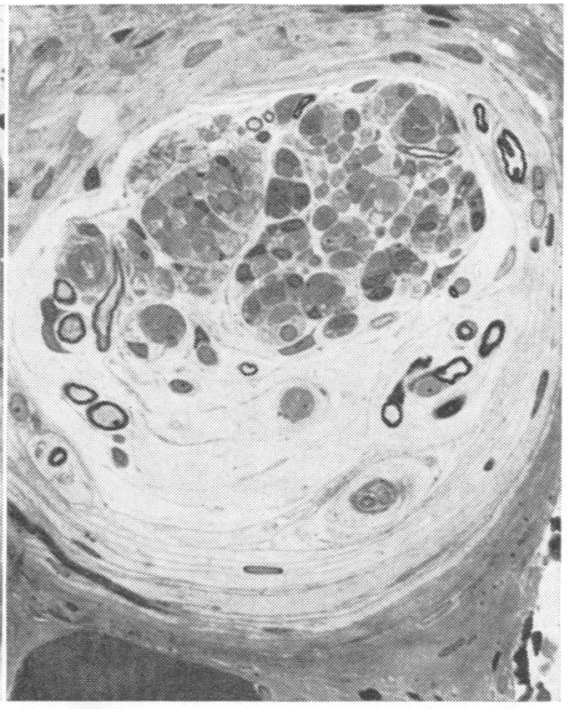

(b)

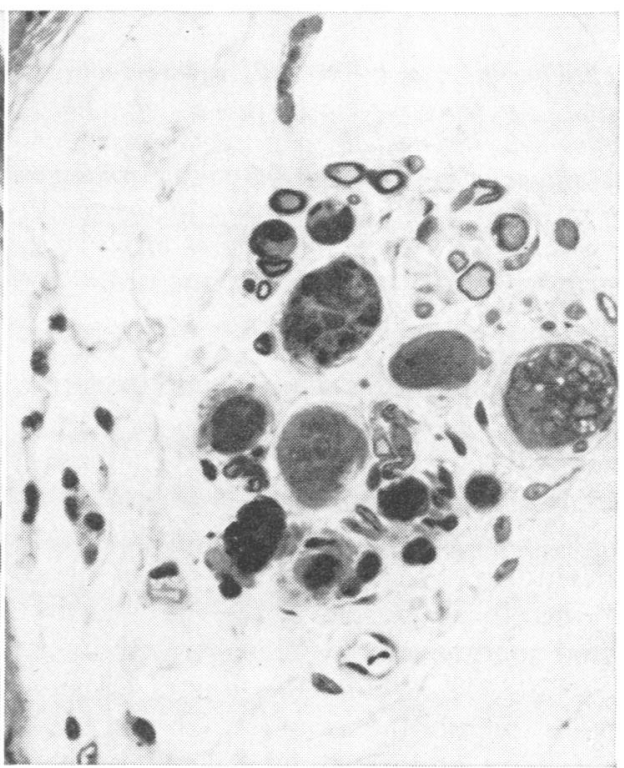

(d)

FIG. $12 \mu \mathrm{m}$ thick, transverse sections of an Araldite-embedded muscle spindle obtained at biopsy. Toluidine blue, $\times 350$. (a) This mid-polar section shows the typical abnormality. The intrafusal muscle fibres are all abnormal. There is marked variation in size and staining intensity of individual fibres. Several myelinated nerve fibres are present. (b) The same spindle sectioned $53 \mu \mathrm{m}$ deeper in the block. Mid-polar section. (c) The same spindle sectioned $162 \mu \mathrm{m}$ from section (a). Two myelinated nerve fibres encircle isolated groups of abnormal intrafusal muscle fibres. The spindle capsule is more prominent and the lumen is larger than in section (b). The section is juxta-equatorial. (d) The same spindle sectioned in its equatorial sensory region, $325 \mu \mathrm{m}$ from section (a). The spindle lumen is larger and there are fewer intrafusal muscle fibres. Several fibres, each containing several nuclei (nuclear bags), have been sectioned through their equatorial sensory regions. 


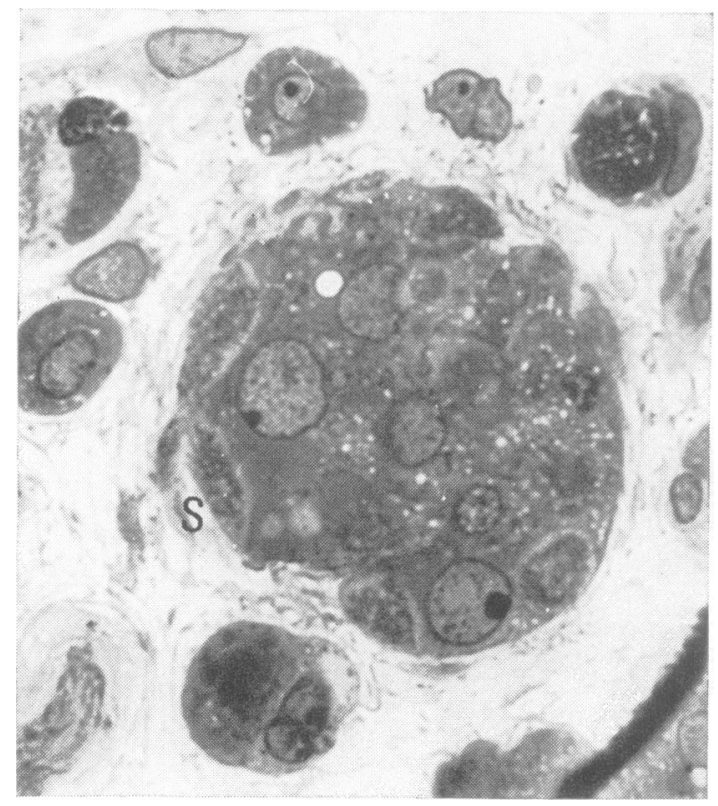

FIG. 2 Toluidine blue, $\times 1400$. This equatorial section was cut 18 um from section 1 (d). The large fibre sectioned through its nuclear bag region contains fewer nuclei than expected, but normal sensory endings (S) can be seen indenting the periphery of the fibre. There are several, additional, small fibres of varying staining intensity.

scattered, single, tiny fibres which sometimes remained discrete for several micra before fusing with a nearby fibre or cluster of fibres. The basement membrane surrounding these isolated single fibres often projected in loose folds into the spindle lumen. Within a cluster, individual abnormal intrafusal muscle fibres, defined by their plasma membranes, were separated by a clear space 130-300 $\AA$ wide. Regions of density and intimate membrane contact (zonula adhaerens) between apposing plasma membranes were common. In some instances a narrow fissure, bounded by partially formed plasma membranes, passing through a region of disrupted myofilaments and swollen, rounded mitochondria seemed to indicate incomplete separation of two fragments of a fibre. Pinocytotic vesicles were prominent beneath the plasma membranes of individual fibres, but very abnormal fibres, for example those containing few myofilaments, contained fewer vesicles (see Fig. 4).

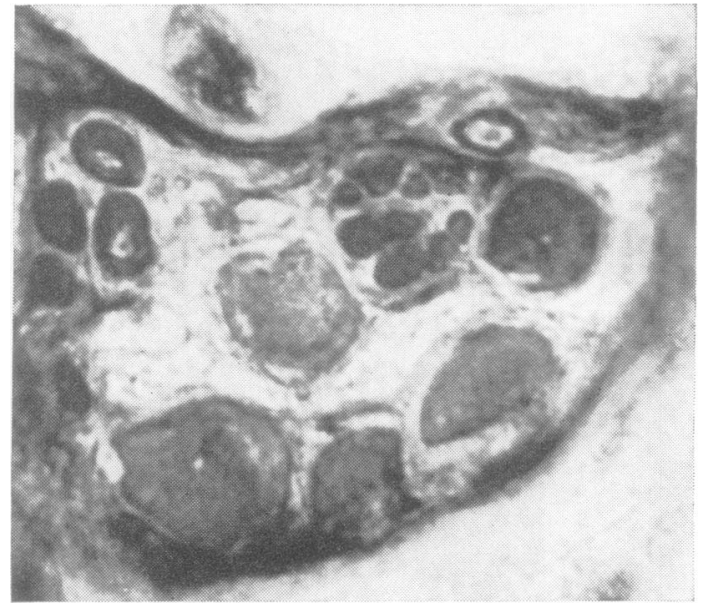

FIG. 3 Myosin ATPase; preincubation at pH 9.5 $\times 900$. This spindle, sectioned in its polar region, contains five normal intrafusal muscle fibres. A sixth fibre, consisting of 11 fragments, occupies an area equivalent to that of an adjacent normal fibre. Several nerve fibres are present in the spindle lumen

There was marked variation in the electron density of adjacent abnormal fibres. This corresponded with the variability of staining intensity observed in the toluidine blue preparations, and was due to degeneration and loss of myofibrils, and to the presence of sarcoplasmic masses. In such fibres myofilaments were misaligned, or present in groups of 5-20 filaments separated from each other by clear sarcoplasm. Some fibres, in which these changes were usually particularly prominent, contained electron-dense rodlets. These rodlets showed the characteristic periodicity of Z-band material (Fig. 5). Clear or dark membrane-bound, lipid vacuoles and lipofuscin granules were common. Several fibres contained myelin bodies, autophagic vacuoles, and other degenerative figures (Fig. 4). The mitochondria and sarcoplasmic reticulum were usually normal, but in the atrophic fragments the few remaining mitochondria were often rounded and swollen; and dilated, short segments of the tubular system were found, usually in close relation to isolated disarrayed myofilaments.

Other fibres contained small groups of well formed myofilaments in a granular cytoplasm, in which polysomes, free ribosomes, glycogen 


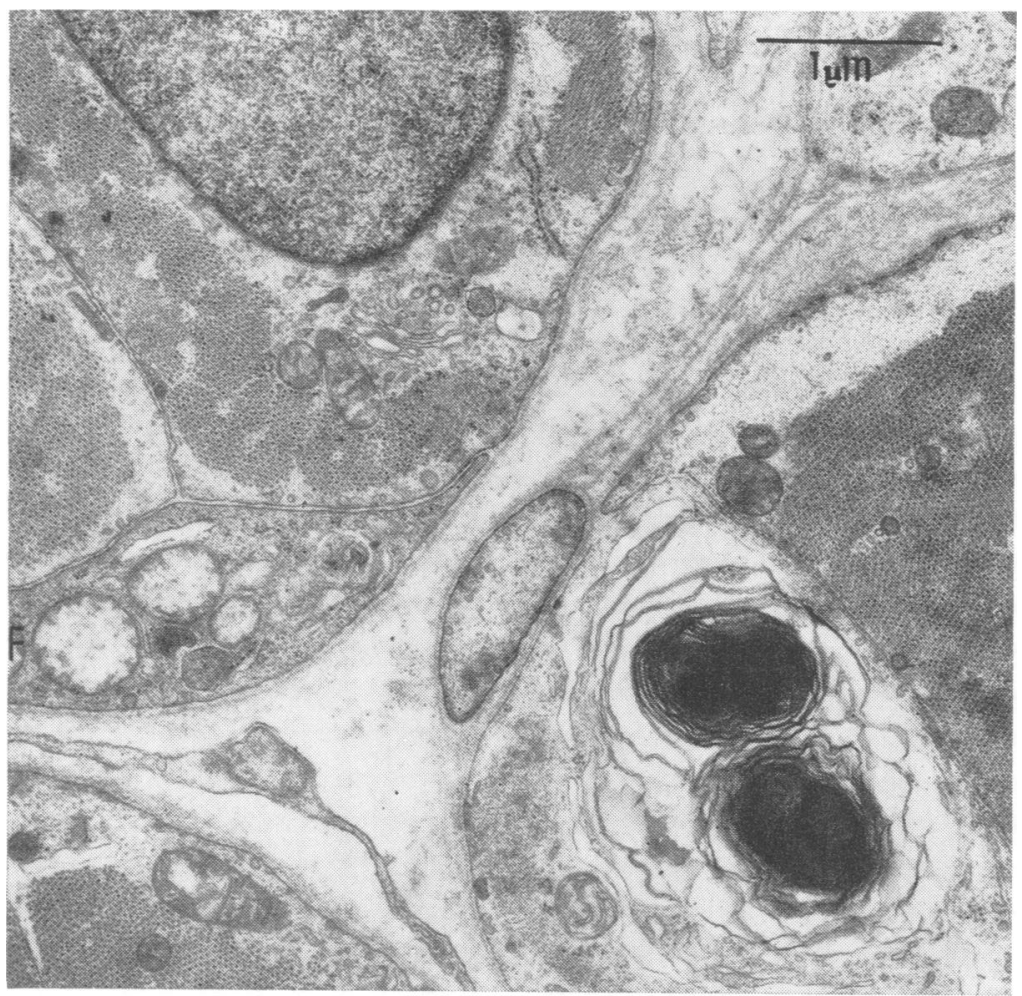

FIG. 4 Electron micrograph, $\times 18000$. One fibre fragment contains densely packed myofibrils. An adjacent fibre contains a lamellated lipid body. Another contains a nucleus, tubules of the Golgi apparatus and rough endoplasmic reticulum. One fibre fragment (F), immediately adjacent to the basement membrane contains granular and vacuolated cytoplasm, and degenerate mitochondrial profiles similar to those described by Hall-Craggs and Lawrence (1970) (see text). Mitochondria in adjacent fibre fragments are normal.

granules, and small mitochondria were abundant. Rough endoplasmic reticulum and a prominent Golgi apparatus were frequent features of these fibres (Fig. 4). In the primary sensory region normal bag (Fig. 6) and chain nuclear aggregations were found. There were prominent clear lipid vacuoles, bound by single membranes.

Myelinated and unmyelinated nerve fibres were found both in the spindle capsule and in close relation to the intrafusal muscle fibres. Sensory nerve fibres were identified by their diameter, and by their termination in typical sensory endings (Smith and Ovalle, 1972), which were invariably normal (see Fig. 6). Motor endings were more difficult to identify since they were small and, apparently, simplified. Plate endings were not identified. These small motor endings were similar to the gamma-trail endings studied in normal cat muscle spindles by Barker et al. (1970), but the fine structure of fusimotor nerve endings has not yet been studied in man and it is not possible, therefore, to state whether these endings were normal or not. There seemed, however, to be fewer motor endings than in normal spindles.

STUDIES OF NECROPSIED MATERIAL Nine spindles were studied in serial transverse section in a single lumbrical muscle. In polar sections each of these spindles contained more than 50 abnormal thin intrafusal muscle fibres but, in their equatorial regions, many of these abnormal fibres fused to form fewer fibres, several of which were of normal diameter, although others remained abnormally small. The larger fibres contained bag or chain aggregations of nuclei, but these nuclei were pyknotic and less numerous than in normal fibres. Basophilia was observed in some of the abnormal intrafusal fibres studied in polar sections.

\section{DISCUSSION}

The striking feature of the spindle abnormality in 


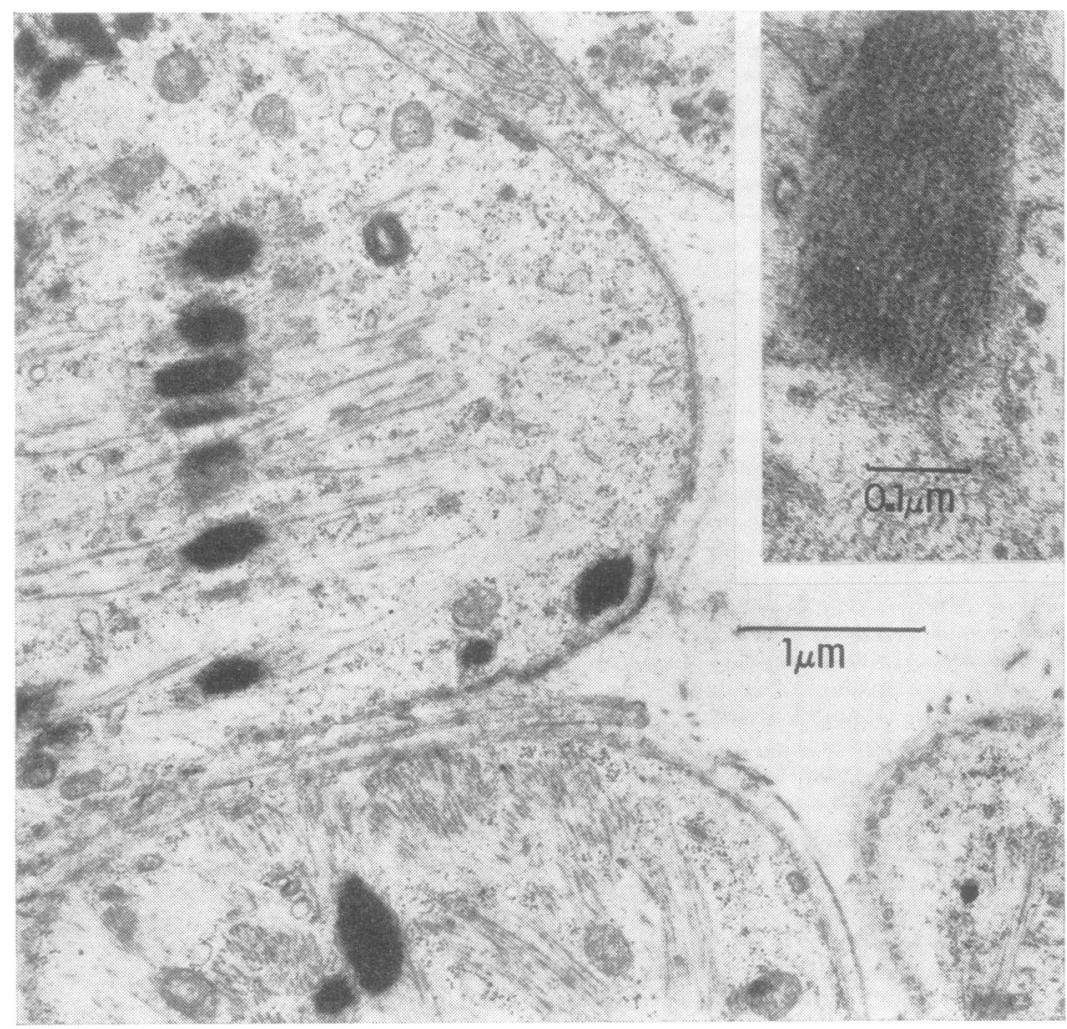

FIG. 5 Electron micrograph, $\times 18000$ (inset, $\times 49$ 900). Prominent rodlike dense bodies, consisting of Z-band material (nemaline bodies) are arranged in close relation to thin, parallel strands of transversely aligned myofilaments.

myotonic dystrophy is the presence, in many spindles, of a large number of very thin intrafusal muscle fibres. In the present work splitting of intrafusal muscle fibres was prominent only in polar sections. The appearance of splitting was found to be the result of a complex process of subdivision and recombination, each subdivided fragment being of very short length. In the equatorial sensory region, and at the extreme tip of the polar region, the intrafusal muscle fibres were almost normal. In the two Aralditeembedded spindles an attempt was made to count the number of abnormal intrafusal muscle fibres at polar, midpolar, and equatorial levels but this was abandoned when it was found that many fragments were too small to allow resolution with the light microscope and others were of such a size and staining intensity that it was difficult to distinguish them from unmyelinated axons. It was felt, therefore, that counting was too unreliable to be useful.
Longitudinal splitting was uncommon in the extrafusal fibres in our Araldite-embedded material, although it has been recognized in extrafusal fibres in previous studies of very advanced cases of myotonic dystrophy (Schröder and Adams, 1968).

In a previous study (Swash, 1972) it was suggested that splitting of intrafusal muscle fibres was preceded by changes in the pattern of the fusimotor innervation. However, the small fragments observed with the electron microscope in the biopsy specimen, described above, would not have been apparent in the teased preparations upon which our earlier work was based and we now consider that it is more likely that these changes in the fusimotor innervation were due to sprouting, as part of an attempt at innervation of these abnormal, fragmented muscle fibres. Furthermore, there is no evidence from studies of the effect of denervation on muscle spindle morphology (Swash and Fox, 1974), that fusi- 


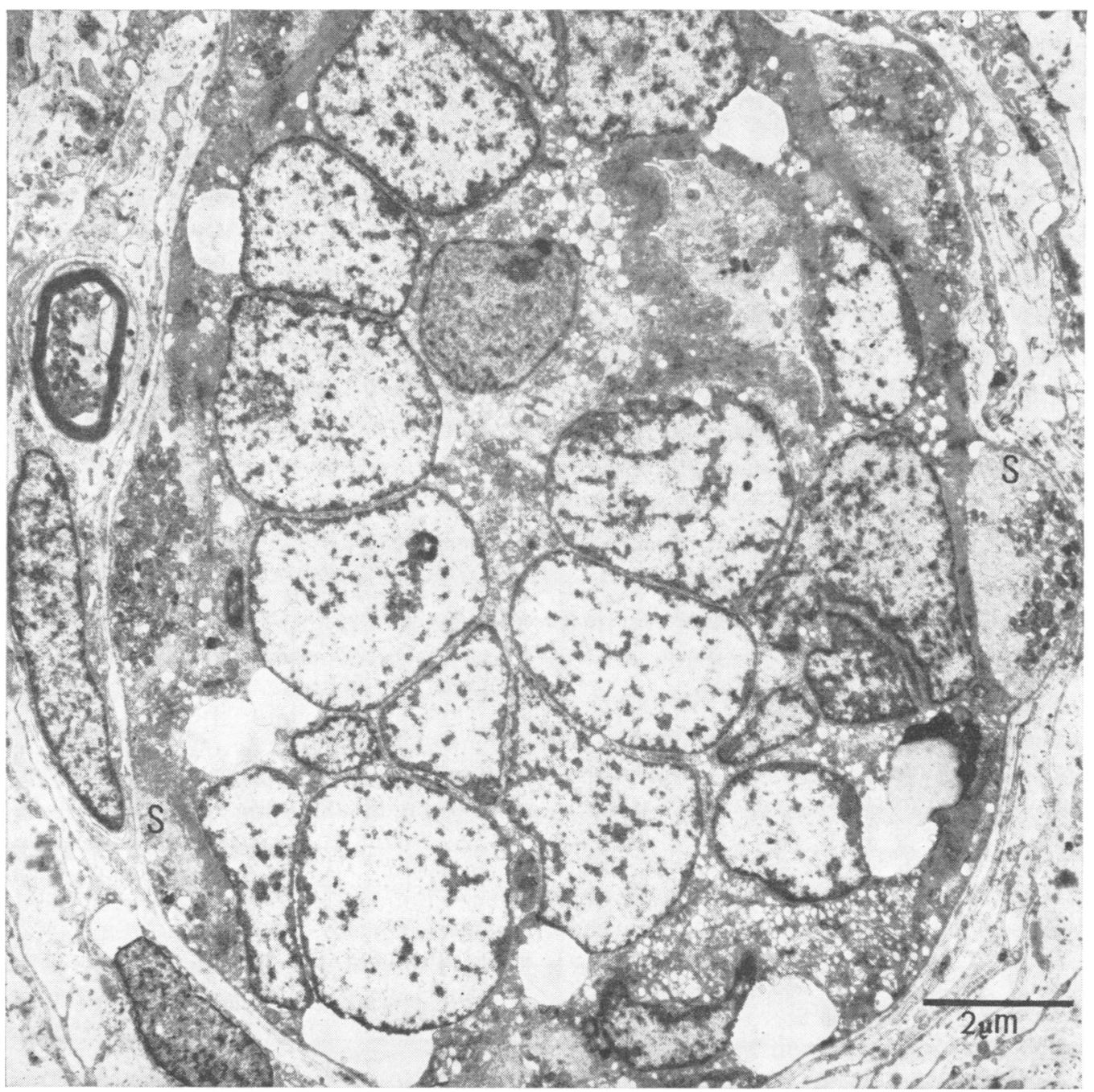

FIG. 6 Electron micrograph, $\times 5$ 400. This fibre, sectioned through its nuclear bag, is surrounded by several sensory endings, containing closely packed mitochondria. The fibre itself is not longitudinally split and its nuclei appear normal. There are numerous single-membrane bounded, clear lipid vacuoles of varying size.

motor denervation would account for the changes found in the intrafusal fibres in myotonic dystrophy.

Isaacs et al. (1973) have studied longitudinal splitting of extrafusal muscle fibres in the dystrophic mouse. They thought that splitting, in these fibres, was due to mechanical stresses. In myotonic dystrophy the severity and complexity of the abnormality of the intrafusal muscle fibres in relation to the minor changes seen in a few extrafusal fibres is difficult to explain on such a simple hypothesis, although it is possible that myotonia and myotonic after-discharges might induce abnormal stresses in the intrafusal fibres. These fibres contract in a localized and graded manner in response to neural stimuli, rather than 
by an all-or-nothing membrane depolarization. Any theory of the pathogenesis of the abnormality must explain the patchy distribution of the spindle abnormality within a given muscle and the observation that it is often severe in muscles that are, otherwise, only moderately abnormal (Swash, 1972).

The possibility that the abnormal intrafusal muscle fibres are new muscle fibres derived from satellite cells (Mauro, 1961), formed as part of an active regenerative process, must also be considered. In extrafusal muscle, regeneration after ischaemic necrosis (Kaspar et al., 1969), and after denervation (Drachman et al., 1969; Hess and Rosner, 1970), occurs from satellite cells formed from, or remaining in, the damaged fibres. In addition, it has been shown, in the rat, that during compensatory hypertrophy satellite structures are formed at the periphery of extrafusal muscle fibres resulting in a muscle containing an increased number of fibres of greatly varying diameter (Hall-Craggs and Lawrence, 1970; James, 1973). Mechanical stress may, therefore, be a stimulus to the formation of satellite structures. Longitudinal splitting, similar in character, but of less complexity, to that found in the intrafusal fibres in myotonic dystrophy, has been observed in rat muscle fibres in the vicinity of experimental crush lesions (Hall-Craggs and Lawrence, 1970). In this work it was suggested that the observed longitudinal division followed damage to the fibres and that it might be a mechanism which allowed rejection of degenerated portions from affected fibres. Regeneration, it was suggested, occurred from viable peripheral portions of affected fibres by satellite cell formation. In myotonic dystrophy individual fragments of intrafusal fibres were separated by their plasma membranes from adjacent fibres, and were arranged in clusters encircled by a single layer of basement membrane. Only some of these abnormal fragments contained sarcolemmal nuclei. These could be regarded as satellite structures (Hess and Rosner, 1970). Those fibres which did not contain nuclei on the other hand, must have been formed by fragmentation or splitting of parent fibres, rather than by a regenerative process.

Some of the abnormal fibres, however, appeared metabolically active. Basophilic fibres were seen in the paraffin-embedded material, and in the electron microscopic preparations active Golgi apparatus and rough endoplasmic reticulum were identified. In others the presence of myelin bodies, lipofuscin, and changes in the myofibrillar pattern suggested that degenerative changes were taking place. The occurrence of rod bodies in some of these fibres is probably not specific (Cape et al., 1970), although Karpati et al. (1971) have suggested that rod bodies may be an indication of a long-standing abnormality of the innervation of muscle. However, in myotonic dystrophy, abnormalities in the fusimotor innervation are probably secondary to fragmentation of the intrafusal fibres.

\section{HYPOTHESIS}

We propose, as a working hypothesis, that the abnormality of intrafusal muscle fibre in myotonic dystrophy may occur as a result of longitudinal splitting, or fragmentation, due to mechanical stresses, perhaps associated with the myotonia, or with myotonic after-discharges. The relative normality of the equatorial sensory region may, perhaps, be explained by the viscous resistance to stretch which is a feature of this region in normal spindles. Splitting may induce the formation of satellite structures, as an attempt at regeneration, and changes consistent with both regeneration and degeneration would then occur in the same group of abnormal intrafusal muscle fibres. Proliferation of fusimotor nerve fibres may represent the attempted innervation of the fragmented, abnormal muscle fibres. In order to test this suggestion, further studies of the fine structure of the polar and equatorial regions of affected intrafusal fibres and of their innervation will be necessary in spindles with varying degrees of abnormality.

This work was supported by the London Hospital Medical College and by a grant for equipment from the Wellcome Trust.

\section{REFERENCES}

Banker, B. Q., and Girvin, J. P. (1971). The ultrastructural features of the mammalian muscle spindle. Journal of Neuropathology and Experimental Neurology, 30, 155-195. Barker, D., Harker, D., Stacey, M. J., and Smith, C. R. (1972). Fusimotor innervation. In Research in Muscle Development and the Muscle Spindle, pp. 227-250. International Congress Series No. 240. Excerpta Medica: Amsterdam. 
Barker, D., Stacey, M. J., and Adal, M. N. (1970). Fusimotor innervation in the cat. Philosophical Transactions of the Royal Society of London, Series B, 258, 315-346.

Cape, C. A., Johnson, W. W., and Pitner, S. E. (1970). Nemaline structures in polymyositis. A nonspecific pathological reaction of skeletal muscles. Neurology (Minneap.), 20, 494-502.

Cooper, S. (1966). Muscle spindles and motor units. In Control and Innervation of Skeletal Muscle, pp. 9-17. Edited by B. L. Andrew. University of St Andrews: Dundee.

Daniel, P. M., and Strich, S. J. (1964). Abnormalities in the muscle spindles in dystrophia myotonica. Neurology (Minneap.), 14, 310-316.

Drachman, D., Wetzel, N., Wasserman, M., and Naito, H. (1969). Experimental denervation of ocular muscles. Archives of Neurology (Chicago), 21, 170-183.

Dubowitz, V., and Brooke, M. H. (1973). Muscle Biopsy: $a$ Modern Approach. Saunders: London.

Hall-Craggs, E. C. B., and Lawrence, C. A. (1970). Longitudinal fibre division in skeletal muscle: a light- and electronmicroscopic study. Zeitschrift für Zellforschung und mikroskopische Anatomie, 109, 481-494.

Hess, A., and Rosner, S. (1970). The satellite cell bud and myoblast in denervated mammalian muscle fibers. American Journal of Anatomy, 129, 21-39.

Isaacs, E. R., Bradley, W. G., and Henderson, G. (1973). Longitudinal fibre splitting in muscular dystrophy; a serial cinematographic study. Journal of Neurology, Neurosurgery, and Psychiatry, 36, 813-819.

James, N. T. (1973). Compensatory hypertrophy in the extensor digitorum longus muscle of the rat. Journal of Anatomy, 116, 57-65.
Karpati, G., Carpenter, S., and Andermann, F. (1971). A new concept of childhood nemaline myopathy. Archives of Neurology (Chicago), 24, 291-304.

Kaspar, U., Wiesmann, U., and Mumenthaler, M. (1969). Necrosis and regeneration of the tibialis anterior muscle in rabbit. I. Histological changes following the ligation of the tibialis anterior artery. Archives of Neurology (Chicago), 21, 363-372.

Landon, D. N. (1966). Electron microscopy of muscle spindles. In Control and Innervation of Skeletal Muscle, pp. 96-111. Edited by B. L. Andrew. University of St Andrews: Dundee.

Mauro, A. (1961). Satellite cell of skeletal muscle fibers. Journal of Biophysical and Biochemical Cytology, 9, 493495.

Schröder, J. M., and Adams, R. D. (1968). The ultrastructural morphology of the muscle fiber in myotonic dystrophy. Acta Neuropathologia, 10, 218-241.

Smith, R. S., and Ovalle, W. K. (1972). The structure and function of intrafusal muscle fibers. In Muscle Biology, vol. 1, pp. 147-227. Edited by R. G. Cassens. Dekker: New York.

Spiro, A. J., and Beilin, R. L. (1969). Human muscle spindle histochemistry. Archives of Neurology (Chicago), 20, 271275.

Swash, M. (1972). The morphology and innervation of the muscle spindle in dystrophia myotonica. Brain, 95, 357368.

Swash, M., and Fox, K. P. (1974). The pathology of the muscle spindle: effect of denervation. Journal of the Neurological Sciences, 22, 1-24. 